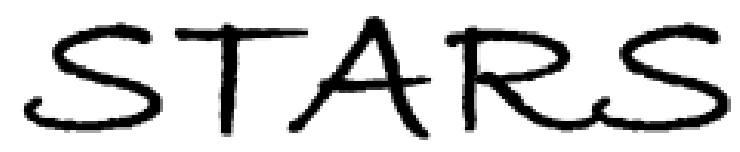

University of Central Florida

STARS

$1-1-2003$

\title{
Investigation of V-Defects and embedded inclusions in InGaN/ GaN multiple quantum wells grown by metalorganic chemical vapor deposition on (0001) sapphire
}

\author{
D. I. Florescu \\ S. M. Ting \\ J. C. Ramer \\ D. S. Lee \\ V. N. Merai
}

Sinednsixtidag enforkd ditti

University of Central Florida Libraries http://library.ucf.edu

This Article is brought to you for free and open access by the Faculty Bibliography at STARS. It has been accepted for inclusion in Faculty Bibliography 2000s by an authorized administrator of STARS. For more information, please contact STARS@ucf.edu.

\section{Recommended Citation}

Florescu, D. I.; Ting, S. M.; Ramer, J. C.; Lee, D. S.; Merai, V. N.; Parkeh, A.; Lu, D.; Armour, E. A.; and Chernyak, L., "Investigation of V-Defects and embedded inclusions in InGaN/GaN multiple quantum wells grown by metalorganic chemical vapor deposition on (0001) sapphire" (2003). Faculty Bibliography 2000s. 3755.

https://stars.library.ucf.edu/facultybib2000/3755

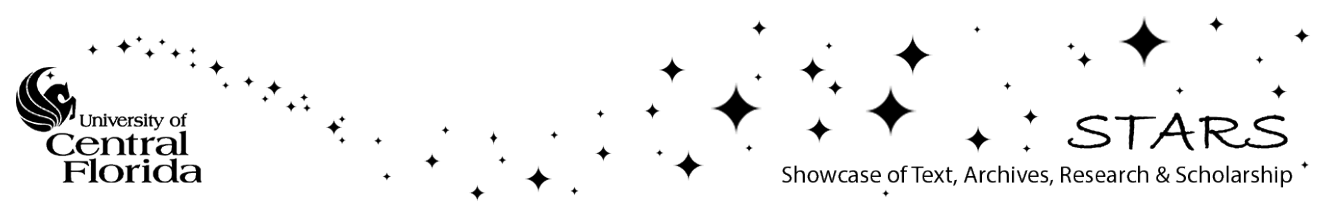




\section{Authors}

D. I. Florescu, S. M. Ting, J. C. Ramer, D. S. Lee, V. N. Merai, A. Parkeh, D. Lu, E. A. Armour, and L. Chernyak 


\section{Investigation of V-Defects and embedded inclusions in InGaN/GaN multiple quantum wells grown by metalorganic chemical vapor deposition on (0001) sapphire}

Cite as: Appl. Phys. Lett. 83, 33 (2003); https://doi.org/10.1063/1.1588370

Submitted: 21 March 2003. Accepted: 30 April 2003 . Published Online: 30 June 2003

D. I. Florescu, S. M. Ting, J. C. Ramer, D. S. Lee, V. N Merai, A. Parkeh, D. Lu, E. A. Armour, and L. Chernyak

\section{ARTICLES YOU MAY BE INTERESTED IN}

Structural origin of V-defects and correlation with localized excitonic centers in InGaN/GaN multiple quantum wells

Applied Physics Letters 72, 692 (1998); https://doi.org/10.1063/1.120844

Morphological evolution of InGaN/GaN quantum-well heterostructures grown by metalorganic chemical vapor deposition

Journal of Applied Physics 94, 1461 (2003); https://doi.org/10.1063/1.1586972

Structure and formation mechanism of V defects in multiple InGaN / GaN quantum well layers Journal of Applied Physics 99, 073505 (2006); https://doi.org/10.1063/1.2180532

\section{Applied Physics Letters}

Mid-IR and THz frequency combs special collection 


\title{
Investigation of V-Defects and embedded inclusions in InGaN/GaN multiple quantum wells grown by metalorganic chemical vapor deposition on (0001) sapphire
}

\author{
D. I. Florescu, ${ }^{\text {a) }}$ S. M. Ting, ${ }^{\text {b) }}$ J. C. Ramer, D. S. Lee, V. N Merai, A. Parkeh, D. Lu, \\ and E. A. Armour \\ EMCORE Corporation, 145 Belmont Drive, Somerset, New Jersey 08873 \\ L. Chernyak \\ Physics Department, University of Central Florida, Florida 32816-2385
}

(Received 21 March 2003; accepted 30 April 2003)

\begin{abstract}
We have examined the nature of V-defects and inclusions embedded within these defects by atomic force microscopy (AFM) and high-resolution scanning electron microscopy (SEM)/ cathodoluminescence (CL) in InGaN/GaN multiple quantum wells (MQWs). To date, indium distribution nonuniformity in the well or $\mathrm{GaN}$ barrier growth temperature have been identified as the main factors responsible for the V-defect occurrence and propagation. Further complicating the matter, inclusions embedded within V-defects originating at the first InGaN-to-GaN interface have been observed under certain growth conditions. Our AFM and high-resolution SEM/CL findings provide evidence that some V-defects occur merely as direct results of barrier temperature growth, and that there are additional V-defects associated with In-rich regions, which act as sinks for further indium segregation during the MQW growth. Both types of V-defects have a tendency of promoting inclusions at low-temperature $\left(800^{\circ} \mathrm{C}\right) \mathrm{GaN}$ barrier growth in an $\mathrm{H}_{2}$-free environment. Localized strain-energy variations associated with the apex of V-defects may be responsible for the inclusion occurrence. Adding $\mathrm{H}_{2}$ during the $\mathrm{GaN}$ barrier growth reduces V-defect formation and suppresses inclusion propagation entirely, rendering a uniform nanoscale CL signal. (C) 2003 American Institute of Physics. [DOI: 10.1063/1.1588370]
\end{abstract}

In recent years, the III-nitride-based alloy system has attracted special attention since high-brightness blue and green light-emitting diodes (LEDs) became commercially available and the room-temperature $\mathrm{CW}$ lasing with this alloy was demonstrated. ${ }^{1}$ Single and/or multiple quantum wells (MQWs) have been used as active layers for blue and green LEDs and laser diodes due to the tunable band-gap energy of InGaN, from 0.7 to $3.4 \mathrm{eV}$ by varying the indium composition. ${ }^{2,3}$ However, despite the remarkable progress in device performance, many problems remain in growing In$\mathrm{GaN}$ epitaxial layers on $\mathrm{GaN}$, particularly with In concentrations higher than $20 \%{ }^{4}$ The role of microstructural and compositional inhomogeneities in the InGaN layers on the optical emission is still unclear. Additionally, InGaN MQW structures often have a so-called V-defect that consists of a threading dislocation terminated by a pit in the shape of an inverted hexagonal pyramid with (1011) sidewalls. ${ }^{5-10}$ To date, indium distribution inhomogeneities in the well material $^{6}$ or $\mathrm{GaN}$ barrier growth temperature ${ }^{9,10}$ have been proposed as the main factors responsible for the V-defect occurrence and propagation. Further complicating the matter, inclusions embedded within $\mathrm{V}$-defects originating at the first InGaN-to-GaN interface have recently been reported under certain growth conditions. ${ }^{11}$

This study reports atomic force microscopy (AFM) and high-resolution scanning electron microscopy (SEM)/ cathodoluminescence (CL) results on $\mathrm{InGaN} / \mathrm{GaN}$ MQWs

\footnotetext{
${ }^{a)}$ Electronic mail: Doru_Florescu@Emcore.com (www.emcore.com).

${ }^{b)}$ Currently at: UMC, Taiwan, ROC.
}

grown by metalorganic chemical vapor deposition (MOVCD) on sapphire (0001). Our findings provide evidence that some V-defects merely occur as direct results of barrier temperature growth, and there are additional V-defects associated with In-rich regions, which act as sinks for further indium segregation during the MQW growth. Both types of V-defects have a tendency of promoting inclusions at low-temperature $\left(800^{\circ} \mathrm{C}\right) \mathrm{GaN}$ barrier growth in an $\mathrm{H}_{2}$-free environment.

Five-period $\mathrm{InGaN} / \mathrm{GaN}$ heterostructures were grown on a $6 \times 2$ in. EMCORE D180 mini-GaNzilla ${ }^{\mathrm{TM}}$ rotating disk reactor on 3- $\mu \mathrm{m} n$-type GaN virtual substrates. Each period consisted of a $30-\AA$ InGaN well with $x_{\mathrm{In}} \sim 25 \%$ (based on growth conditions) followed by a $120-\AA$ GaN barrier. Two types of samples were produced. For each of the GaN barriers in the heterostructure the growth was performed either in an $\mathrm{H}_{2}$-free environment or with the addition of $5 \operatorname{sim} \mathrm{H}_{2}$. Several runs were verified for reproducibility and two representative samples for the $\mathrm{H}_{2}$-free MQW (sample A) and $\mathrm{H}_{2}$-MQW (sample B) were selected for our studies.

AFM was performed using a Digital Instruments DI3100 in tapping mode. Typically, a $5 \times 5-\mu \mathrm{m}^{2}$ AFM map with lateral resolution of about $10 \mathrm{~nm}$ (limited by the tip radius) was acquired at $1-\mathrm{Hz}$ scan rate.

A Philips XL30 SEM integrated with a Gatan MonoCL3 CL system was used for electron microscopy. The emitted radiation was analyzed using a single grating (1200 lines/ $\mathrm{mm}$, blazed at $500 \mathrm{~nm}$ ) and a Hamamatsu photomultiplier tube, sensitive in the 185-850-nm range. Panoramic CL imaging was carried out using a $10-\mathrm{keV}$ beam. Wave-dependent 

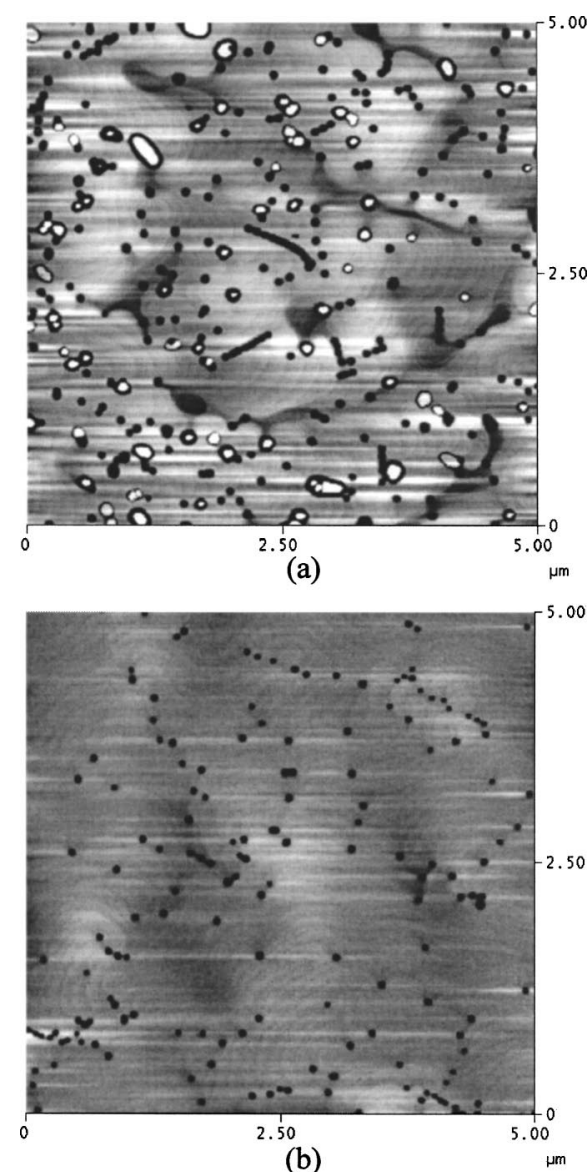

(b)

FIG. $1.5 \times 5-\mu \mathrm{m}^{2}$ AFM scans of the (a) $\mathrm{H}_{2}$-free MQW (sample A) and (b) $\mathrm{H}_{2}$-MQW (sample B).

CL spectra were acquired at $5-\mathrm{keV}$ accelerating voltage (320 $000 \times$ magnification), giving an electron range of about $70 \mathrm{~nm}$ in the samples.

The AFM images in Fig. 1 show the $5 \times 5-\mu \mathrm{m}^{2}$ scans of the two MQW samples. Sample A, which had its GaN barriers grown $\mathrm{H}_{2}$-free is pictured in Fig. 1(a) and sample $\mathrm{B}$, for which the $\mathrm{GaN}$ barriers were grown with the addition of 5 $\operatorname{slm} \mathrm{H}_{2}$ is shown in Fig. 1(b). Striking morphological differences are noticeable. A higher density of pits with a wider range of pit diameters is noted for sample A [Fig. 1(a)] when compared to sample B [Fig. 1(b)]. In addition, the typical inclusions embedded within the pits, which are characteristic for $\mathrm{H}_{2}$-free $\mathrm{GaN}$ barrier growth at low temperature $\left(800^{\circ} \mathrm{C}\right)$ in $\mathrm{InGaN} / \mathrm{GaN}$ heterostructures are observed for sample $\mathrm{A}$ as bright, irregular white features. The inclusion-free defect density in Fig. 1(a) is in the $8-9 \times 10^{8} / \mathrm{cm}^{2}$ range, with the average defect diameter of about 80 to $100 \mathrm{~nm}$. The defect density in the case of inclusion-filled pits is about 2-3 $\times 10^{8} / \mathrm{cm}^{2}$ with diameters in the $100-400-\mathrm{nm}$ range. Inclusion heights up to $120 \AA$ are observed. A reduced density of pits in the $6-7 \times 10^{8} / \mathrm{cm}^{2}$ range with a narrower pit diameter distribution between 50 and $100 \mathrm{~nm}$ is noted for sample B [Fig. 1(b)]. The rms/dZ range values for sample A and B are $2.62 \mathrm{~nm} / 47.87 \mathrm{~nm}$ and $1.05 \mathrm{~nm} / 25.60 \mathrm{~nm}$, respectively.

High-resolution secondary electrons (SE) and CL are shown in Figs. 2(a) and 2(b), respectively. Bright (spot 1) and dark (spot 2) inclusion-filled pits are noticeable and are indicated by arrows. A defect-free area (spot 3) was also considered. The CL spectrum was acquired at the locations

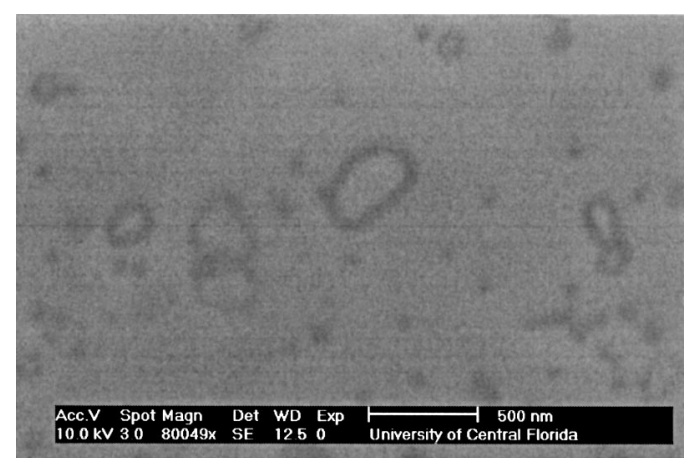

(a)

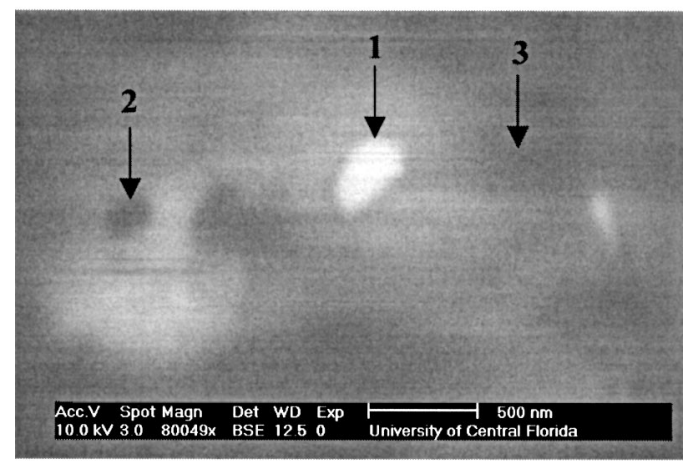

(b)

FIG. 2. High-resolution SEM images of sample A with (a) SE and (b) CL views for the area indicated by the dashed rectangle in Fig. 2(a). The arrows in (b) define spots 1,2, and 3, where CL spectra were acquired. The horizontal bar is $500 \mathrm{~nm}$ at approximately $80000 \times$.

indicated in Fig. 3(b) using a higher SEM magnification (not shown). A 9-nm redshift was found between the spots marked " 1 " compared to " 2 " and " 3 ," with the wavelength/ intensity values being $513 \mathrm{~nm} / 14800$ a.u. and $504 \mathrm{~nm} / 13800$ a.u., respectively. The full width at half-maximum (FWHM) was about $30 \mathrm{~nm}$ for all three locations.

Figure 3 shows high-resolution SEM/CL images of sample B; that is, the $\mathrm{H}_{2}-\mathrm{MQW}$. Similar features to the AFM results in Fig. 1(b) are observed in Fig. 3(a). Micro-CL reveals uniform brightness with no apparent pit effect, as shown in Fig. 3(b). The arrow marks a random defect-free location (spot 4). The CL spectrum acquired using a higher SEM magnification at this location shows an emission wavelength/intensity of $496 \mathrm{~nm} / 12000$ a.u. (not shown). The CL FWHM was about $35 \mathrm{~nm}$.

Based on the AFM and high-resolution SEM/CL observations of this study, V-defect formation in InGaN/GaN MQWs clearly cannot by treated as the effect of either indium nonuniformities or barrier growth temperature alone. The high-resolution CL image of Fig. 2(b) seems to be indicating that some $\mathrm{V}$-defects occur merely as direct results of low $\left(800^{\circ} \mathrm{C}\right)$ barrier temperature growth, since no enhanced CL signal (i.e., brighter, longer wavelength) is observed when compared to defect-free areas. In addition, there are V-defects that may be associated with In-rich regions, where an enhanced CL signal is found. A 9-nm CL wavelength redshift and higher intensity are noted when spot 1 is compared to spots 2 and 3 in Fig. 2(b). This may be an indication of the fact that the V-defect at spot 1 acts as a sink for further indium segregation during subsequent InGaN/GaN QW layer 


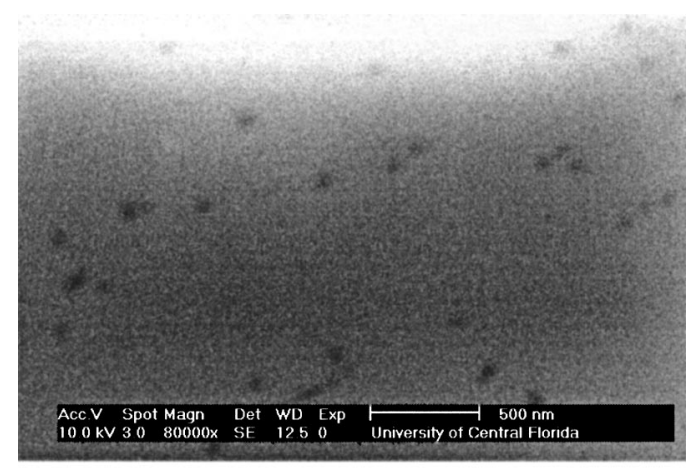

(a)

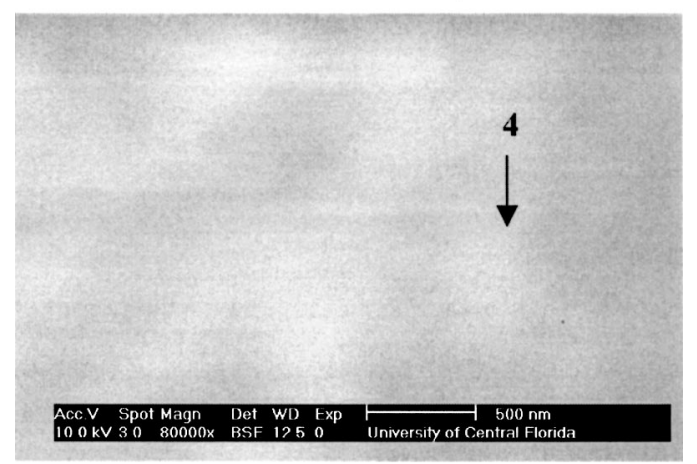

(b)

FIG. 3. High-resolution SEM images of sample B with (a) SE and (b) CL views. The arrow in (b) defines spot 4 where CL spectrum was acquired. The horizontal bar is $500 \mathrm{~nm}$ at approximately $80000 \times$.

growth. The defect at spot 2 displays a similar CL signal with the defect-free area at spot 3 , indicative of similar indium concentrations. By supplying $\mathrm{H}_{2}$ during the barrier growth, the V-defect density is reduced by about a factor of 2 , the inclusion occurrence and propagation is suppressed. The 8-nm CL wavelength blueshift, intensity decrease, and broader FWHM at spot 4 in Fig. 3(b) compared to spots 2 and 3 in Fig. 2(b) appear to be a clear indication of an overall reduction of the In mole fraction and, at the same time, a more uniform In incorporation for the QWs grown at the same barrier temperature under $\mathrm{H}_{2}$. The V-defects observed in Fig. 3(a) are probably the result of barrier growth temperature only, since a uniform CL signal is displayed as seen in Fig. 3(b).

We observe that some of the V-defects have a tendency of promoting inclusions in an $\mathrm{H}_{2}$-free environment. Both types of defects, namely ones believed to be generated as a results of the barrier growth temperature [spot 2 in Fig. 2(b)] and with higher indium contents [spot 1 in Fig. 2(b)] display this behavior. When an inclusion originates and develops within a V-defect, it disturbs its propagation with the addition of subsequent QW layers. A variety of shapes and heights are noticeable in Fig. 1(a) for the inclusions embedded within pits. Cremades et al. suggested that the built in strain in InGaN layers determines the preferential incorporation of In atoms to the alloy in regions where the strain is relaxed via the formation of defects. ${ }^{12}$ However, these localized strain-energy variations associated with the areas around the apex of the V-defects at the first InGaN-to-GaN interface may play a more significant role than indium segregation in the case of inclusion occurrence, since they are observed for V-defects with both higher and similar to defect-free area indium content. Moreover, the first InGaN well for the samples used in this study is highly strained, since the critical thickness of InGaN $\left(x_{\mathrm{In}} \sim 20 \%\right)$ epilayers grown on $\mathrm{GaN}$ was estimated to be at least $4000 \AA{ }^{3}{ }^{3}$ Strain relaxation of low and high indium composition InGaN/GaN MQWs was previously reported. ${ }^{13}$ The extent to which strain relaxation (if present) will influence the inclusion propagation throughout the MQW structure is unknown at this time, but we speculate that inclusion occurrence will not be affected since they are believed to be originating at the first InGaN-to-GaN interface, where strain effects are strongly present.

We have used AFM and high-resolution SEM/CL to investigate $\mathrm{V}$-defect and embedded inclusion formation in InGaN/GaN MQWs grown by MOVCD on sapphire (0001). Our findings provide evidence that some V-defects occur merely as direct results of barrier temperature growth, and there are additional V-defects associated with In-rich regions, which act as sinks for further indium segregation during the MQW growth. Both types of V-defects have a tendency of promoting inclusions at low temperature $\left(800^{\circ} \mathrm{C}\right) \mathrm{GaN}$ barrier growth in an $\mathrm{H}_{2}$-free environment. Localized strainenergy variations associated with the apex of V-defects at the first InGaN-to-GaN interface may be responsible for the inclusion occurrence.

Research at UCF is supported in part by the National Science Foundation (ECS\#0216055).

${ }^{1}$ S. J. Pearton, J. C. Zolper, R. J. Shul, and F. Ren, J. Appl. Phys. 86, 1 (1999).

${ }^{2}$ T. Matsuoka, H. Okamoto, M. Nakao, H. Harima, and E. Kurimoto, Appl. Phys. Lett. 81, 1246 (2002).

${ }^{3}$ S. C. Jain, M. Willander, J. Narayan, and R. V. Overstraeten, J. Appl. Phys. 87, 965 (2000).

${ }^{4}$ N. A. El-Masry, E. L. Piner, S. X. Liu, and S. M. Bedair, Appl. Phys. Lett. 72, 40 (1998).

${ }^{5}$ X. H. Wu, C. R. Elsaas, A. Abare, M. Mack, S. Keller, P. M. Petroff, S. P. DenBaars, J. S. Speck, and S. J. Rosner, Appl. Phys. Lett. 72, 692 (1998).

${ }^{6}$ I.-H. Kim, H.-S. Park, Y.-J. Park, and T. Kim, Appl. Phys. Lett. 73, 1634 (1998).

${ }^{7}$ J. E. Northrup, L. T. Romano, and J. Neugebauer, Appl. Phys. Lett. 74, 2319 (1999).

${ }^{8}$ N. Duxbury, U. Bangert, P. Dawson, E. J. Thrush, W. Van der Stricht, K. Jacobs, and I. Moerman, Appl. Phys. Lett. 76, 1600 (2000).

${ }^{9}$ F. Scholz, J. Off, E. Fehrenbacher, O. Gfrorer, and B. Brockt, Phys. Status Solidi A 180, 315 (2000).

${ }^{10}$ J. T. Kobayashi, N. P. Kobayashi, and P. D. Dapkus, 1998 March Meeting of The American Physical Society, Los Angeles, 16-20 March 1998.

${ }^{11}$ S. M. Ting, J. C. Ramer, D. I. Florescu, V. N. Merai, B. E. Albert, A. Parekh, D. S. Lee, D. Lu, D. V. Christini, and E. A. Armour, J. Appl. Phys. (in press).

${ }^{12}$ A. Cremades, V. Navarro, J. Piqueras, A. P. Lima, O. Ambacher, and M. Stutzmann, J. Appl. Phys. 90, 4868 (2001).

${ }^{13}$ T. Wang, D. Nakagawa, J. Wang, T. Sugahara, and S. Sakai, Appl. Phys. Lett. 73, 3571 (1998). 